\title{
Impact du nucléaire sur la santé et l'environnement en situation non accidentelle
}

Entretien croisé(a) avec Claude Stéphan, physicien, CNRS, et Pierre Barbey, biologiste, Université de Caen

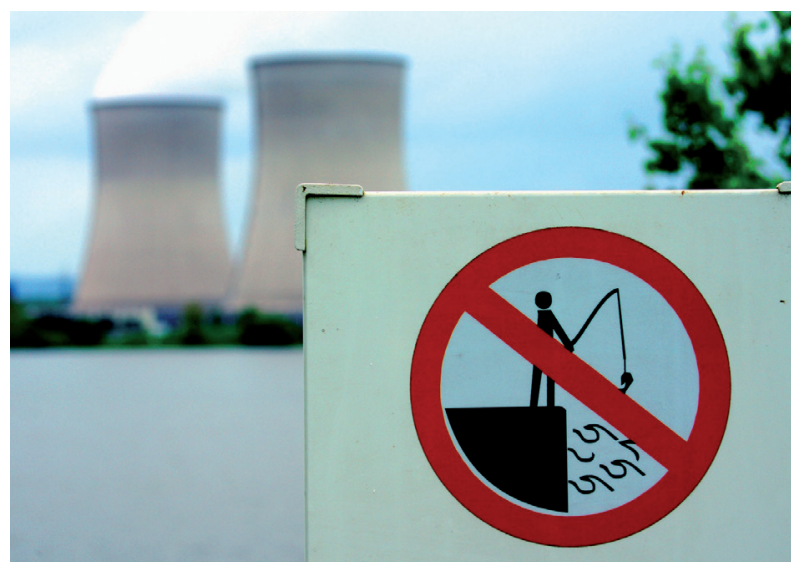

Quel est l'impact du fonctionnement d'une centrale nucléaire sur l'environnement, les terres et les populations avoisinantes? Quelles sont les phases industrielles de la filière, de l'extraction du minerai à l'exploitation des centrales, qui comportent les effets sensibles les plus controversés?

Deux chercheurs répondent à nos questions en exprimant des points de vue opposés.

\section{De l'extraction du minerai au réacteur}

Lorsqu'on pense à l'impact sur l'environnement de l'exploitation de la filière nucléaire civile, on pense tout de suite au réacteur, ses rejets et à son éventuelle dangerosité sur les populations voisines. Cependant, il est important de considérer aussi la question en amont et de se pencher sur la vie du combustible nucléaire avant qu'il ne soit inséré dans un réacteur. En effet, bien que quelques milliers de tonnes ${ }^{(b)}$ d'uranium naturel suffisent pour alimenter tous les réacteurs du parc français pendant un an, les procédures d'extraction du minerai comportent des contraintes et ce n'est pas sans conséquence sur l'environnement et les populations.

Le premier impact est de nature radiologique : en effet, l'uranium est légèrement radioactif et la décroissance de sa radioactivité conduit entre autres à la formation de radon, un gaz radioactif présent en quantité dans les mines : une mine d'uranium a dix à cent fois la radioactivité naturelle ${ }^{(c)}$. Le principe de l'extraction de l'uranium reposant sur une action mécanique (concassage et broyage) suivie d'un traitement chimique, toutes ces substances radioactives sont également libérées de la texture minérale qui les confinait et deviennent facilement mobilisables avec le passage de l'eau.

L'extraction de l'uranium sur le territoire français, commencée en 1949 et essentiellement terminée dans les années 1990, a vu l'exploitation d'environ 250 sites, répartis dans 27 départements, qui ont amené à la production de 76000 tonnes d'uranium. Si la totalité de l'uranium utilisé pour la consommation française est aujourd'hui importée (principalement du Niger, du Canada, d'Australie et du Kazakhstan), il n'en a pas toujours été ainsi et l'impact n'en est pas pour autant amoindri. D'une part, le choix d'importer la matière première de pays étrangers ne fait que déplacer hors du territoire français l'impact de l'industrie minière. D'autre part, même dans les mines françaises aujourd'hui fermées, il subsiste quelques activités de contrôle ${ }^{(\mathrm{d})}$.
Claude Stéphan reconnait que les conditions du développement des premières mines et les pratiques d'exploitation à l'époque ont alimenté l'opinion défavorable sur l'extraction d'uranium. La perception du public est largement fondée sur les impacts sanitaires et environnementaux découlant de pratiques appliquées à une époque révolue où il n'y avait pas vraiment de réglementation. Effectivement, dans les débuts, les travailleurs étaient exposés à des niveaux de rayonnement aujourd'hui considérés comme dangereux, pouvant provoquer, en particulier, des cancers du poumon.

C'est principalement, après la fermeture de la plupart des sites miniers français, la mobilisation d'associations relative à des situations de pollution radioactive ou de gestion des déchets miniers et des terrils qui ont conduit les pouvoirs publics à réagir. D’une part, en engageant un important travail d'expertise pluraliste entre 2006 et 2010 et en instituant localement des «Comités de suivi des anciens sites miniers ", mais aussi en renforçant l'encadrement réglementaire concernant la gestion de ces anciens sites. 
Les conditions de gestion d'un site d'extraction sont aujourd'hui réglementées, avec l'introduction d'un régime d'inspections et de contrôles réglementaires et administratifs effectués sous l'autorité des préfets par les Directions Régionales de l'Environnement. Ce dispositif permet de réduire la possibilité que des objectifs politiques ou économiques influencent les décisions réglementaires. On est donc passé d'une planification pratiquement inexistante à des procédés de traitement des effluents à plusieurs étapes et des systèmes de gestion conçus spécialement pour la filière.

Si l'impact radiologique semble donc aujourd'hui plutôt maitrisé dans les anciens sites miniers français, aujourd'hui fermés, Pierre Barbey rappelle que l'impact sur l'environnement est également de nature chimique. À commencer par l'uranium lui-même qui, s'il est certes un élément radioactif, est tout d'abord un élément chimique très toxique. C'est d'ailleurs sur la base de sa toxicité chimique que l'Organisation Mondiale de la Santé (OMS) a fixé une limite en concentration en uranium de $15 \mu \mathrm{g} / \mathrm{l}$ pour les eaux potables. Il faut noter cependant que l'établissement de limites et recommandations basées sur la toxicité n'est pas une affaire facile. Une des difficultés de cet exercice, s'agissant des anciens sites de mines d'uranium, est que les substances toxiques extraites ou produites sont d'origine naturelle et que l'action anthropique s'ajoute à un bruit de fond naturel, lui-même fluctuant. Pour apprécier l'impact dosimétrique et aider à la fixation de limites, l'OMS a choisi d'estimer les transferts des polluants toxiques dans l'environnement et de prendre en compte l'ensemble des voies d'atteinte de l'homme selon des scénarios d'exposition s'appuyant sur des modes de vie.

Un dernier élément important à souligner quant à l'impact environnemental des sites miniers est que leur réhabilitation après le démantèlement des installations est une activité industrielle à part entière mais complexe. L'État français a confié à Areva (désormais Orano) la gestion et la surveillance des anciens sites miniers français, y compris ceux qu'il n'a pas exploités. Le réaménagement des sites vise en priorité à limiter l'impact résiduel des activités passées : sécuriser les installations pour le public, assurer la surveillance radiologique et environnementale, assurer le traitement des eaux pour les sites qui le nécessitent. Une centaine de spécialistes sont ainsi mobilisés chaque année pour réaliser près de 7000 analyses environnementales, géologiques, radiologiques et sanitaires.

Après l'extraction de l'uranium, vient la problématique de son transport. Les difficultés principales liées au transport de substances radioactives sont le risque d'inhalation ou d'ingestion de particules radioactives, le risque d'irradiation externe, et la contamination de l'environnement. Environ 10\% des colis nucléaires transportés en France sont en lien avec l'industrie électronucléaire et cela représente environ 19000 transports annuels, pour 114000 colis $^{(\mathrm{e})}$. Les déplacements des marchandises dangereuses par transport routier, ferroviaire ou maritime sont réglementés par les autorités nationales. Le colis doit apporter par lui-même une protection suffisante pour éviter les conséquences d'un accident sur la population et l'environnement. La substance radioactive est enfermée dans des futs en acier étanches chargés dans des conteneurs certifiés par l'Organisation Internationale de Normalisation (ISO) avec le marquage et la signalisation appropriée. Par conséquent, en situation non accidentelle, le transport des matières nucléaires ne semble pas présenter un impact spécifique sur l'environnement et les populations.

\section{Du réacteur jusqu'aux déchets}

En fonctionnement non accidentel, l'impact d'une centrale nucléaire sur l'environnement est essentiellement lié aux rejets thermiques, chimiques et radioactifs qui exposent les travailleurs du nucléaire et le public. Les rejets industriels liés à la filière nucléaire ne sont pas très différents de ceux produits par toute autre centrale thermique. Pour autant, l'après-Tchernobyl a vu la création, sous la pression de l'opinion publique, d'instances de contrôle indépendantes, l'Autorité de sureté nucléaire (ASN) et l'Institut de radioprotection et de sureté nucléaire (IRSN), dans le but de gérer l'ensemble des risques nucléaires (électronucléaire, médecine, etc.). L'ASN participe à l'élaboration de règles relatives à l'énergie nucléaire, et surveille leur bonne exécution, alors que l'IRSN coordonne des recherches en liaison avec la sureté nucléaire et garde mémoire des retours d'expérience effectués dans les centrales. Avec l'exploitant, ces deux institutions participent ainsi aux visites décennales destinées à évaluer si une centrale peut ou non continuer à être exploitée, à effectuer des contrôles et à confirmer le niveau de sureté de l'installation. Enfin, l'ASN définit les limites règlementaires concernant tous les rejets qu'une centrale nucléaire est autorisée à émettre en exploitation.

En particulier, l'exploitation des centrales nucléaires entraine notamment une production d'eaux et de gaz usés et de chaleur. En France, il existe des limites règlementaires pour éviter un réchauffement local trop important de la source froide (fleuve, rivière, mer) car l'eau prélevée est restituée à une température légèrement supérieure à sa température de prélèvement. En conséquence, la production doit être diminuée ou suspendue si l'eau restituée est trop chaude par rapport au débit de la source froide (effet de dilution).

Pour ce qui est des rejets radioactifs, Claude Stéphan rappelle qu'au cours des vingt dernières années, le parc nucléaire d'EDF a divisé par un facteur supérieur à 100 l'activité de ses rejets radioactifs hors gaz rares, tritium et carbone 14. Dans ce dernier cas, l'émission dans l'environnement de cet isotope est extrêmement faible et, comme en outre elle se fait essentiellement sous une forme (le méthane) qui n'est pas assimilable par les végétaux, elle ne constitue qu'environ $1 \%$ du bruit de fond moyen mesuré. L'impact épidémiologique sur les populations vivant à proximité des centrales nucléaires françaises est considéré comme non significatif. Cependant, de nombreuses associations de riverains surveillent avec attention et inquiétude l'évolution des niveaux de radioactivité dans les sols et les eaux des nappes phréatiques ${ }^{(\mathrm{f})}$.

La dose annuelle d'irradiation maximale autorisée pour les travailleurs du nucléaire est de 20 millisievert $^{(\mathrm{g})}$, soit un peu plus de quatre fois la dose d'irradiation naturelle. Dans les faits, le niveau de radiation subi est bien inférieur et le nombre de dépassements de ce seuil se réduit chaque année. Cette dose d'irradiation est-elle dangereuse ? La question reste ouverte. 
Les études disponibles ne décèlent aucun effet pour des doses inférieures à 100 millisievert, soit qu'il n'en existe pas, soit que la puissance statistique des enquêtes ait été insuffisante pour les détecter ${ }^{(h)}$. Le public au voisinage immédiat d'une centrale nucléaire en reçoit dix mille fois moins, soit 0,002 millisievert par an, ce qui est négligeable par rapport à ce qu'elle reçoit par la radioactivité naturelle (notamment celle du radon) et suite aux examens radiologiques.

Le cas du plutonium, et plus généralement de l'option de retraitement de combustibles usés issus de la filière d'EDF mais aussi de clients étrangers, est plus controversé. Pierre Barbey souligne que, comparativement aux autres activités de la filière, le retraitement est une étape particulièrement polluante. Les travaux d'inventaire menés dans la seconde moitié des années 1990 par un groupe d'experts pluraliste, le Groupe Radioécologie Nord-Cotentin (GRNC), ont conduit à identifier 73 éléments radioactifs (hors éléments de courte demi-vie) issus des opérations de retraitement, soit le double des radioéléments déclarés par l'opérateur à cette époque. Le site de La Hague dispose actuellement d'autorisations de rejets de polluants radioactifs et chimiques dans l'environnement délivrées par l'ASN en tant qu'autorité compétente. Mises en service en 1966, les installations de retraitement de La Hague ont engendré des rejets qui n'ont cessé de croitre, du fait de la montée en activité, jusqu'au milieu des années 1980. C'est à ce moment que l'exploitant a mis en place une nouvelle gestion des effluents et, depuis, une baisse progressive de ces rejets est observée. Pour autant, ce nouveau système ne concerne pas certains radioéléments non retenus (tritium, gaz rares...) qui continuent à progresser corrélativement au tonnage retraité. À noter que, contrairement aux centrales nucléaires, le carbone 14 est ici principalement rejeté sous forme de $\mathrm{CO}_{2}$, assimilable par les végétaux, et qu'il est le principal contributeur à la dose reçue par les populations.

La fin de vie du combustible usé, et donc la gestion des déchets produits, est aujourd'hui un sujet qui n'a pas de solution simple ${ }^{(\mathrm{i})}$. Claude Stéphan rappelle d'abord que les fragments de fission représentent presque toute la radioactivité produite et l'immense majorité d'entre eux a une demi-vie qui n'excède pas 30 ans. Ce type de déchets est entreposé au Centre de Stockage de l'Aube (CSA), dont est chargée l'Agence nationale pour la gestion des déchets radioactifs (ANDRA), dans des futs métalliques contenus à leur tour dans des surconteneurs en béton. La radioactivité de ces matières, dites de faible et moyenne activité à vie courte, sera réduite d'un facteur 1000 après environ 300 ans, et leur entreposage en surface est considéré aujourd'hui comme une solution limitant sensiblement l'impact sur l'environnement.

En revanche, le reste du combustible usé, qui constitue les déchets ultimes (autres produits de fission, et actinides mineurs tels que l'americium, le neptunium, ...), de moyenne ou haute activité à vie longue, pose des défis technologiques plus importants. Ces déchets sont aujourd'hui vitrifiés, c'est-à-dire mélangés à une matrice de verre, matière connue pour sa bonne tenue à la chaleur et aux radiations, et entreposés en attente d'une décision sur le stockage de long terme. La solution aujourd'hui à l'étude en France prévoit le stockage en couche profonde, de l'ordre de $500 \mathrm{~m}$, dans le centre industriel de stockage géologique (CIGEO), objet d'un débat actuel, qui requiert une stabilité géologique et sismique à l'échelle des dizaines de milliers d'années.

Pierre Barbey fait observer que cette filière d'élimination des déchets, comme d'autres, n'existe aujourd'hui qu'à l'état de projet. Actuellement il n'existe que deux centres de stockage de surface : outre celui de l'Aube, le premier centre de stockage historique est celui de la Manche (CSM), objet de nombreuses polémiques, du fait de sa situation en zone marécageuse et régulièrement inondable ; le confinement de sa radioactivité est critiqué par des associations ${ }^{(j)}$, et il est en phase de fermeture. Le Centre Industriel de Regroupement, d'Entreposage et de Stockage (CIRES), également dans l'Aube et géré par l'ANDRA, est dédié aux déchets de très faible activité.

En résumé, l'impact des mines d'uranium en France semble largement diminué par l'arrêt de leur exploitation, sans supprimer le besoin d'un contrôle constant de la pollution qui en a résulté, tout en déplaçant les impacts dans les pays actuellement producteurs. Le transport des matières fissiles semble sous contrôle. Les rejets par les centrales en activité sont considérés comme non significatifs. La pollution liée au retraitement des combustibles est en diminution mais reste mesurable. Quant au stockage des déchets radioactifs de moyenne ou haute activité à vie longue, c'est un problème d'importance majeure qui est encore débattu.

a.Voir dans ce dossier, le préambule aux entretiens croisés, par F. Graner et S. M. Panebianco (p. 18)

b. À titre de comparaison, cette masse équivaut à seulement quelques pourcents du chargement d'un seul superpétrolier.

c. L'uranium 238 lui-même est présent depuis la formation de la Terre et sa demi-vie est de l'ordre de 4,5 milliards d'années, l'uranium 235 de 700 millions d'années, ce qui veut dire que leur activité naturelle est faible. Certains descendants sont de courte demi-vie : les principaux radioéléments problématiques pour l'homme et l'environnement sont le radium 226, le polonium 210 et le plomb 210. À titre de comparaison, l'ordre de grandeur de la radioactivité naturelle est de $100 \mathrm{~Bq} / \mathrm{kg}$ pour des roches basaltiques ou sédimentaires, $1000 \mathrm{~Bq} / \mathrm{kg}$ pour des roches granitiques. $\mathrm{La}$ radioactivité des roches résiduelles (dites "stériles ") de mines d'uranium est typiquement de $10000 \mathrm{~Bq} / \mathrm{kg}$, celle des résidus de traitement des minerais d'uranium est de $500000 \mathrm{~Bq} / \mathrm{kg}$, ce qui étonnamment est comparable aux résidus des centrales électriques à lignite en service en Allemagne ou en Pologne. Les minerais d'uranium eux-mêmes ont une activité typique de $1000000 \mathrm{~Bq} / \mathrm{kg}$.

d. Une mine n'étant pas considérée comme une installation nucléaire de base (INB), elle n'est pas soumise à une procédure de démantèlement.

e. Les autres colis nucléaires sont essentiellement des sources nucléaires à usage industriel, comme la stérilisation des aliments (60\%), ou à usage médical $(30 \%)$. Au total, ces colis nucléaires représentent quelques pourcents de l'ensemble des colis de matières dangereuses. Source : ASN

f. Voir dans ce dossier l'article de D. Boilley (p. 24).

g. Sievert : unité évaluant l'impact des rayonnements sur l'humain.

h. De très petites doses peuvent certes endommager un brin d'ADN, mais pas les deux, et dans ce cas la cellule peut le réparer correctement. Une dose plus forte a statistiquement plus de probabilité de couper les deux brins, elle a donc des effets bien plus importants.

i. Sur la question des déchets, voir plusieurs articles dans ce dossier, en particulier ceux de J.-Y. Le Déaut (p. 13) et de B. Romagnan (p. 14).

j.Voir les critiques portées par le réseau «Sortir du nucléaire ", l'ACRO ou Greenpeace : par exemple le rapport ACRO de 2006 révisé en 2009, www.acro.eu.org/Archives/CSM_GP09.pdf 